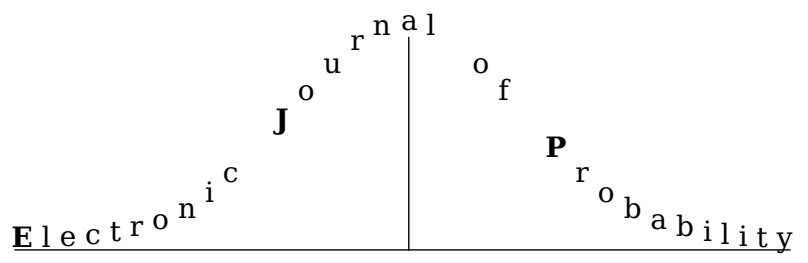

Electron. J. Probab. 24 (2019), no. 104, 1-23.

ISSN: 1083-6489 https://doi.org/10.1214/19-EJP364

\title{
Gradient Gibbs measures for the SOS model with countable values on a Cayley tree
}

\author{
Florian Henning* \\ Christof Külske ${ }^{\dagger}$ \\ Utkir A. Rozikov ${ }^{\S}$ \\ Arnaud Le $\mathrm{Ny}^{\ddagger}$
}

\begin{abstract}
We consider an SOS (solid-on-solid) model, with spin values from the set of all integers, on a Cayley tree of order $k \geq 2$ and are interested in tree-automorphism invariant gradient Gibbs measures (GGMs) of the model. Such a measure corresponds to a boundary law (a function defined on vertices of the Cayley tree) satisfying a functional equation. In the ferromagnetic SOS case on the binary tree we find up to five solutions to a class of period- 4 height-periodic boundary law equations (in particular, some period-2 height-periodic ones). We show that these boundary laws define up to four distinct GGMs. Moreover, we construct some period-3 height-periodic boundary laws on the Cayley tree of arbitrary order $k \geq 2$, which define GGMs different from the 4-periodic ones.
\end{abstract}

Keywords: SOS model; Cayley tree; Gibbs measure; tree-indexed Markov chain; gradient Gibbs measures; boundary law.

AMS MSC 2010: Primary 82B26, Secondary 60K35.

Submitted to EJP on February 14, 2019, final version accepted on September 18, 2019.

\section{Introduction}

We consider models where an infinite-volume spin-configuration $\omega$ is a function from the vertices of the Cayley tree to a local configuration space $E \subseteq \mathbb{Z}$.

A solid-on-solid (SOS) model is a spin system with spins taking values in (a subset of) the integers, and formal Hamiltonian

$$
H(\omega)=-J \sum_{\{x, y\}}\left|\omega_{x}-\omega_{y}\right|,
$$

where $J \in \mathbb{R}$ is a coupling constant. As usual, $\{x, y\}$ denotes a pair of nearest neighbour vertices.

${ }^{*}$ Ruhr-University of Bochum, Germany. E-mail: Florian. Henning@ruhr-uni-bochum.de

${ }^{\dagger}$ Ruhr-University of Bochum, Germany. E-mail: Christof. Kuelske@ruhr-uni-bochum. de

${ }^{\ddagger}$ Université Paris-Est, France. E-mail: arnaud. le-ny@u-pec. fr

§Institute of Mathematics Tashkent, Uzbekistan. E-mail: rozikovu@yandex.ru 
For the local configuration space we consider in the present paper the full set $E:=\mathbb{Z}$. The model can be considered as a generalization of the Ising model, which corresponds to $E=\{-1,1\}$, or a less symmetric variant of the Potts model with non-compact state space. SOS-models on the cubic lattice were analyzed in [16] where an analogue of the so-called Dinaburg-Mazel-Sinai theory was developed. Besides interesting phase transitions in these models, the attention to them is motivated by applications, in particular in the theory of communication networks; see, e.g., [13], [18].

SOS models in $\mathbb{Z}^{d}$ with $E=\mathbb{Z}$ have also been used as simplified discrete interface models which should approximate the behaviour of a Dobrushin-state in an Ising model when the underlying graph is $\mathbb{Z}^{d+1}$, and $d \geq 1$. There is the issue of possible nonexistence of any Gibbs measure in the case of such unbounded spins, in particular in the additional presence of disorder. (Height-)periodic probability measures on the single-site spin space $\mathbb{Z}$ can not exist. When a proper Gibbs-measure exists, therefore it cannot be height-periodic. Proper Gibbs measures for the discrete-height $\mathbb{Z}$-valued SOS model exist in dimensions $d \geq 2$, when the inverse temperature $\beta$ is sufficiently large. This can be seen by a Peierls argument. For continuous-height models, by contrast, proper Gibbs measures do not exist in $d \leq 2$, while gradient Gibbs measures do exist also in $d=2$ [11]. Adding local disorder to the discrete SOS-models spoils the existence of proper (random) Gibbs measures in dimensions $d=2$ [5]. In higher dimensions $d \geq 3$ at 'small disorder' the existence of proper (random) Gibbs measures holds [4], [3]. This is proved using a renormalization group method in the spirit of Bricmont-Kupiainen [6] which is an iterated expansion method. For continuous-height disordered models on the lattice even (random) GGMs fail to exist in $d=2$ [10] while they do exist in $d \geq 3$, for potentials bounded above and below by a square [7].

In the present paper we show that on the Cayley tree there are several treeautomorph-ism invariant gradient Gibbs measures. In contrast to tree-automorphism Gibbs measures which e.g. do not exist on the Cayley tree of dimension one due to the normalisability condition on probability measures, gradient Gibbs measures always exist.

For even more background on gradient Gibbs measures on the lattice, also in the case of real valued state space, we refer to [23], [1], [8] and [2].

If we restrict the local spin space from the set of all integers $\mathbb{Z}$ to a finite number, we arrive at the $m$-state SOS-model, which is a Potts-type model, but with an SOSinteraction. Compared to the Potts model, the $m$-state SOS model has less symmetry: The full symmetry of the Hamiltonian under joint permutation of the spin values is reduced to the mirror symmetry, which is the invariance of the model under the map $\omega_{i} \mapsto m-\omega_{i}$ on the local spin space. Therefore one expects a more diverse structure of phases.

To the best of our knowledge, the first paper devoted to the SOS model on the Cayley tree is [19]. In [19] the case of arbitrary $m \geq 1$ is treated and a vector-valued functional equation for possible boundary laws of the model is obtained. Recall that each solution to this functional equation determines a splitting Gibbs measure (SGM), in other words a tree-indexed Markov chain which is also a Gibbs measure. Such measures can be obtained by propagating spin values along the edges of the tree, from any site singled out to be the root to the outside, with a transition matrix depending on initial Hamiltonian and the boundary law solution. In particular the homogeneous (site-independent) boundary laws then define translation-invariant (TI) SGMs. For a recent investigation of the influence of weakly non-local perturbations in the interaction to the structure of Gibbs measures, see [2] in the context of the Ising model.

Also the symmetry (or absence of symmetry) of the Gibbs measures under spin reflection is seen in terms of the corresponding boundary law. For SOS models some 
TISGMs which are symmetric have already been studied in the particular case $m=2$ in [19], and $m=3$ in [20]. In [14], for $m=2$, a detailed description of TISGMs (symmetric and non-symmetric ones) is given: it is shown the uniqueness in the case of antiferromagnetic interactions, and existence of up to seven TISGMs in the case of ferromagnetic interactions. See also [21] for more details about SOS models on trees.

In the situation of an unbounded local spin space the normalisability condition given in [25] (which is needed to construct a SGM, in other words a tree-indexed Markov chain, from a given boundary law solution) is not automatically satisfied anymore. In this paper we are interested in the class of (spatially homogeneous/ tree-automorphism invariant) height-periodic boundary laws to tree-automorphism invariant spin-translation/heightshift invariant potentials whose elements violate this normalisability condition. Here, a spatially homogeneous period- $q$ height-periodic boundary law is a $q$-periodic function on the local state space $\mathbb{Z}$. Although the procedure of constructing a Gibbs measures from boundary laws described in [25] can not be applied to elements of that class, we are still able to assign a tree-automorphism invariant gradient Gibbs measure (GGM) on the space of gradient configurations to each such spatially homogeneous heightperiodic boundary law, compare [15]. This motivates the study of spatially homogeneous height-periodic boundary laws as useful finite-dimensional objects which are are easier to handle than the non-periodic ones required to fulfill the normalisability condition. Gradient Gibbs measures describe height differences, Gibbs measures describe absolute heights. Each Gibbs measure defines a gradient Gibbs measure, but the converse is not true, which is a phenomenon that is well-known from the lattice. Some more explanation will be given in the following sections. The main goal of this paper then consists in the description of a class of boundary solutions for the $\mathbb{Z}$-valued SOS-model which have periods of 2, 3 and 4 with respect to shift in the height direction on the local state space $\mathbb{Z}$, and their associated GGMs. Our motivation is to present closed-form solutions, prove identifiability, and demonstrate the richness of transitions even inside these families. While the expectation of height-differences is always zero in all of these gradient states, the states really do differ. This is precisely the conclusion of our discussion of their identifiability, where we construct families of events which allow to distinguish them. To see boundary law-dependence, we also refer to Section 5.3 ('Correlation decay is governed by the fuzzy chain') in [15]. The general picture which emerges is that as the inverse temperature becomes large, more and more gradient states appear. This is shown by the examples of period up to four, but similar phenomena should appear for higher periods. Higher periods are not analyzed in the present paper but this is certainly an interesting topic.

The paper is organized as follows. In Section 2 we first present the preliminaries of our model. Section 3 then contains a summary on the notion of GGMs on trees and their construction from homogeneous height-periodic boundary laws. For further details see [15]. The main part, Section 4, is devoted to the description of a set of homogeneous period-2, 3 and 4 height-periodic boundary laws for the SOS-model on $\mathbb{Z}$. Solving the associated boundary law equations for the period- 2 and the period- 4 height-periodic case on the binary tree we prove that, depending on the system parameters, this set contains one up to five elements, yet the number of distinct GGMs assigned to them will turn out to be at most four. In the last subsection we construct GGMs for period-3 height-periodic boundary laws on the $k$-regular tree for arbitrary $k \geq 2$.

\section{Preliminaries}

Cayley tree. The Cayley tree $\Gamma^{k}$ of order $k \geq 1$ (or $k$-regular tree) is an infinite tree, i.e. a locally finite connected graph without cycles, such that exactly $k+1$ edges 
originate from each vertex. Let $\Gamma^{k}=(V, L)$ where $V$ is the set of vertices and $L$ the set of edges. Two vertices $x, y \in V$ are called nearest neighbours if there exists an edge $l \in L$ connecting them. We will use the notation $l=\{x, y\}$. A collection of nearest neighbour pairs $\left\{x, x_{1}\right\},\left\{x_{1}, x_{2}\right\}, \ldots,\left\{x_{d-1}, y\right\}$ is called a path from $x$ to $y$. The distance $d(x, y)$ on the Cayley tree is the number of edges of the shortest path from $x$ to $y$.

Furthermore, for any $\Lambda \subset V$ we define its outer boundary as

$$
\partial \Lambda:=\{x \notin \Lambda: d(x, y)=1 \text { for some } y \in \Lambda\} .
$$

SOS model. We consider a model where the spin takes values in the set of all integer numbers $\mathbb{Z}:=\{\ldots,-1,0,1, \ldots\}$, and is assigned to the vertices of the tree. A (height) configuration $\omega$ on $V$ is then defined as a function $x \in V \mapsto \omega_{x} \in \mathbb{Z}$; the set of all height configurations is $\Omega:=\mathbb{Z}^{V}$. Take the power set $2^{\mathbb{Z}}$ as measurable structure on $\mathbb{Z}$ and then endow $\Omega$ with the product $\sigma$-algebra $\mathcal{F}:=\sigma\left\{\omega_{i} \mid i \in V\right\}$ where $\sigma_{i}: \Omega \rightarrow \mathbb{Z}$ denotes the projection on the $i$ th coordinate. We also sometimes consider more general finite subsets $\Lambda$ of the tree and we write $\mathcal{S}$ for the set of all those finite subtrees. Recall here that the (formal) Hamiltonian of the SOS model is

$$
H(\omega)=-J \sum_{\{x, y\} \in L}\left|\omega_{x}-\omega_{y}\right|,
$$

where $J \in \mathbb{R}$ is a constant which we will set to 1 (incorporated in the inverse temperature $\beta$ ) in the following. As defined above, $\{x, y\}$ denotes nearest neighbour vertices.

Note that the above Hamiltonian is invariant under the spin-translation/height-shift $t:(t \omega)_{i}=\omega_{i}+1$. (It is given by a gradient interaction potential in the terminology of [15].) This suggests reducing the complexity of the configuration space by considering gradient configurations instead of height configurations as will be explained in the following section.

\section{Gradient Gibbs measures and an infinite system of functional equations}

Gradient configurations: Let the Cayley tree be called $\Gamma^{k}$. We may induce an orientation on $\Gamma^{k}$ relative to an arbitrary site $\rho$ (which we may call the root) by calling an edge $\langle x, y\rangle$ oriented iff it points away from the $\rho$. More precisely, the set of oriented edges is defined by

$$
\vec{L}:=\overrightarrow{L_{\rho}}:=\{\langle x, y\rangle \in L: d(\rho, y)=d(\rho, x)+1\} .
$$

Note that the oriented graph $(V, \vec{L})$ also possesses all tree-properties, namely connectedness and absence of loops.

For any height configuration $\omega=(\omega(x))_{x \in V} \in \mathbb{Z}^{V}$ and $b=\langle x, y\rangle \in \vec{L}$ the height difference along the edge $b$ is given by $\nabla \omega_{b}=\omega_{y}-\omega_{x}$ and we also call $\nabla \omega$ the gradient field of $\omega$. The gradient spin variables are now defined by $\eta_{\langle x, y\rangle}=\omega_{y}-\omega_{x}$ for each $\langle x, y\rangle \in \vec{L}$. Let us denote the space of gradient configurations by $\Omega^{\nabla}=\mathbb{Z}^{\vec{L}}$. Note that in contrast to the notion used in [23] for the lattice $\mathbb{Z}^{d}$, the gradient configurations defined above are indexed by the oriented edges of the tree and not by its vertices. Equip the integers $\mathbb{Z}$ with the power set as measurable structure. Having done this, the measurable structure on the space $\Omega^{\nabla}$ is given by the product $\sigma$-algebra $\mathcal{F}^{\nabla}:=\sigma\left(\left\{\eta_{b} \mid b \in \vec{L}\right\}\right)$. Clearly $\nabla:(\Omega, \mathcal{F}) \rightarrow\left(\Omega^{\nabla}, \mathcal{F}^{\nabla}\right)$ then becomes a measurable map.

For any fixed site $x \in V$ and given spin value $\omega_{x} \in \mathbb{Z}$, each gradient configuration $\zeta \in \Omega^{\nabla}$ (uniquely) determines a height configuration by the measurable map

$$
\varphi_{x, \omega_{x}}:\left\{\begin{array}{l}
\Omega^{\nabla} \rightarrow \Omega \\
\left(\varphi_{x, \omega_{x}}(\zeta)\right)_{y}=\omega_{x}+\sum_{b \in \Gamma(x, y)} \zeta_{b},
\end{array}\right.
$$


where $\Gamma(x, y)$ is the unique path from $x$ to $y$. From this we get the following two statements:

1. The linear map $\nabla: \mathbb{Z}^{V} \rightarrow \mathbb{Z}^{\vec{L}}$ is surjective and

2. The kernel of $\nabla$ is given by the spatially homogeneous configurations.

Therefore we have the identification

$$
\Omega^{\nabla}=\mathbb{Z}^{\vec{L}}=\mathbb{Z}^{V} / \mathbb{Z}
$$

Here, $=$ is meant in the sense of isomorphy between Abelian groups. Endowing $\mathbb{Z}^{V} / \mathbb{Z}$ with the final $\sigma$-algebra generated by the respective coset projection, we can also regard this isomorphism as an isomorphism between measurable spaces due to the measurability of the maps $\varphi_{x, \omega_{x}}$ and $\nabla$.

Note that statement (1) above relies on the absence of loops in trees. For gradient configurations on lattices in more than one dimension a further plaquette condition is needed (see [11]). In contrast to this, the following statement (2) is based on connectedness of the tree. Therefore, for any finite subtree $\Lambda \in \mathcal{S}$, the isomorphism (3.2) between measurable spaces restricts to an isomorphism between $\mathbb{Z}^{\Lambda} / \mathbb{Z}$ and $\mathbb{Z}^{\{b \in \vec{L} \mid b \subset \Lambda\}}$, where the sets are endowed with the respective final and product $\sigma$-algebra.

Further note that for any $w \in V$ the bijection

$$
\left\{\begin{array}{l}
\mathbb{Z}^{V} \rightarrow \mathbb{Z}^{\vec{L}} \times \mathbb{Z} \\
\omega=\left(\omega_{x}\right)_{x \in V} \mapsto\left(\nabla \omega, \omega_{w}\right)
\end{array}\right.
$$

is an isomorphism with respect to the product $\sigma$-algebra on $\mathbb{Z}^{\vec{L}} \times \mathbb{Z}$, where the inverse map is given by (3.1). In the following, this will allow us to easily identify any measure on $\mathbb{Z}^{V}$ with its push-forward on the space $\mathbb{Z}^{\vec{L}} \times \mathbb{Z}$.

Gibbs measure: Recall that the set of height configurations $\Omega:=\mathbb{Z}^{V}$ was endowed with the product $\sigma$-algebra $\otimes_{i \in V} 2^{\mathbb{Z}}$, where $2^{\mathbb{Z}}$ denotes the power set of $\mathbb{Z}$. Then, for any $\Lambda \subset V$, consider the coordinate projection map $\sigma_{\Lambda}: \mathbb{Z}^{V} \rightarrow \mathbb{Z}^{\Lambda}$ and the $\sigma$-algebra $\mathcal{F}_{\Lambda}:=\sigma\left(\sigma_{\Lambda}\right)$ of cylinder sets on $\mathbb{Z}^{V}$ generated by the map $\sigma_{\Lambda}$.

Now we are ready to define Gibbs measures on the space of height-configurations for the model (2.1) on a Cayley tree. Let $\nu=\{\nu(i)>0, i \in \mathbb{Z}\}$ be a $\sigma$-finite positive fixed a-priori measure, which in the following we will always assume to be the counting measure.

Gibbs measures are built within the DLR framework by describing conditional probabilities w.r.t. the outside of finite sets, where a boundary condition is frozen. One introduces a so-called Gibbsian specification $\gamma$ so that any Gibbs measure $\mu \in \mathcal{G}(\gamma)$ specified by $\gamma$ verifies

$$
\mu\left(A \mid \mathcal{F}_{\Lambda^{c}}\right)=\gamma_{\Lambda}(A \mid \cdot) \quad \mu-\text { a.s. }
$$

for all $\Lambda \in \mathcal{S}$ and $A \in \mathcal{F}$. The Gibbsian specification associated to a potential $\Phi$ is given at any inverse temperature $\beta>0$, for any boundary condition $\omega \in \Omega$ as

$$
\gamma_{\Lambda}(A \mid \omega)=\frac{1}{Z_{\Lambda}^{\beta, \Phi}} \int e^{-\beta H_{\Lambda}^{\Phi}\left(\sigma_{\Lambda} \omega_{\Lambda^{c}}\right)} \mathbf{1}_{A}\left(\sigma_{\Lambda} \omega_{\Lambda^{c}}\right) \nu^{\otimes \Lambda}\left(d \sigma_{\Lambda}\right),
$$

where the partition function $Z_{\Lambda}^{\beta, \Phi}$ - that has to be non-null and convergent in this countable infinite state-space context (this means that $\Phi$ is $\nu$-admissible in the terminology of [12]) - is the standard normalization whose logarithm is often related to pressure or free energy. 
In our SOS-model on the Cayley tree, $\Phi$ is the unbounded nearest-neighbour potential with $\Phi_{\{x, y\}}\left(\omega_{x}, \omega_{y}\right)=\left|\omega_{x}-\omega_{y}\right|$ and $\Phi_{\{x\}} \equiv 0$, so $\gamma$ is a Markov specification in the sense that

$$
\gamma_{\Lambda}\left(\omega_{\Lambda}=\zeta \mid \cdot\right) \text { is } \mathcal{F}_{\partial \Lambda} \text {-measurable for all } \Lambda \subset V \text { and } \zeta \in \mathbb{Z}^{\Lambda} \text {. }
$$

In order to build up gradient specifications from the Gibbsian specifications defined above, we need to consider the following: Due to the absence of loops in trees, for any finite subgraph $\Lambda \subset \mathbb{Z}$, the complement $\Lambda^{c}$ is not connected, but consists of at least two connected components where each of these contains at least one element of $\partial \Lambda$. This means that the gradient field outside $\Lambda$ does not contain any information on the relative height of the boundary $\partial \Lambda$ (which is to be understood as an element of $\mathbb{Z}^{\partial \Lambda} / \mathbb{Z}$ ). More precisely, let $c c\left(\Lambda^{c}\right)$ denote the number of connected components in $\Lambda^{c}$ and note that $2 \leq c c\left(\Lambda^{c}\right) \leq|\partial \Lambda|$.

Applying (3.1) to each connected component, an analogue to (3.2) becomes

$$
\mathbb{Z}^{\Lambda^{c}} / \mathbb{Z}=\mathbb{Z}^{\left\{b \in \vec{L} \mid b \subset \Lambda^{c}\right\}} \times\left(\mathbb{Z}^{c c\left(\Lambda^{c}\right)} / \mathbb{Z}\right) \subset \mathbb{Z}^{\left\{b \in \vec{L} \mid b \subset \Lambda^{c}\right\}} \times\left(\mathbb{Z}^{\partial \Lambda} / \mathbb{Z}\right)
$$

where "=" is in the sense of isomorphy between measurable spaces. For any $\eta \in$ $\Omega^{\nabla}=\mathbb{Z}^{V} / \mathbb{Z}$, let $[\eta]_{\partial \Lambda} \in \mathbb{Z}^{\partial \Lambda} / \mathbb{Z}$ denote the image of $\eta$ under the coordinate projection $\mathbb{Z}^{V} / \mathbb{Z} \rightarrow \mathbb{Z}^{\partial \Lambda} / \mathbb{Z}$ with the latter set endowed with the final $\sigma$-algebra generated by the coset projection. Set

$$
\mathcal{F}_{\Lambda}^{\nabla}:=\sigma\left(\left(\eta_{b}\right)_{b \subset \Lambda^{c}}\right) \subset \mathcal{T}_{\Lambda}^{\nabla}:=\sigma\left(\left(\eta_{b}\right)_{b \subset \Lambda^{c}},[\eta]_{\partial \Lambda}\right)
$$

Then $\mathcal{T}_{\Lambda}^{\nabla}$ contains all information on the gradient spin variables outside $\Lambda$ and also information on the relative height of the boundary $\partial \Lambda$. By (3.7) we have that for any event $A \in \mathcal{F}^{\nabla}$ the $\mathcal{F}_{\Lambda^{c}}$-measurable function $\gamma_{\Lambda}(A \mid \cdot)$ is also measurable with respect to $\mathcal{T}_{\Lambda}^{\nabla}$, but in general not with respect to $\mathcal{F}_{\Lambda}^{\nabla}$. These observations lead to the following:

Definition 3.1. The gradient Gibbs specification is defined as the family of probability kernels $\left(\gamma_{\Lambda}^{\prime}\right)_{\Lambda \subset \subset V}$ from $\left(\Omega^{\nabla}, \mathcal{T}_{\Lambda}^{\nabla}\right)$ to $\left(\Omega^{\nabla}, \mathcal{F}^{\nabla}\right)$ such that

$$
\int F(\rho) \gamma_{\Lambda}^{\prime}(d \rho \mid \zeta)=\int F(\nabla \varphi) \gamma_{\Lambda}(d \varphi \mid \omega)
$$

for all bounded $\mathcal{F}^{\nabla}$-measurable functions $F$, where $\omega \in \Omega$ is any height-configuration with $\nabla \omega=\zeta$.

Using the sigma-algebra $\mathcal{T}_{\Lambda}^{\nabla}$, this is now a proper and consistent family of probability kernels, i.e.

$$
\gamma_{\Lambda}^{\prime}(A \mid \zeta)=\mathbf{1}_{A}(\zeta)
$$

for every $A \in \mathcal{T}_{\Lambda}^{\nabla}$ and $\gamma_{\Delta}^{\prime} \gamma_{\Lambda}^{\prime}=\gamma_{\Delta}^{\prime}$ for any finite volumes $\Lambda, \Delta \subset V$ with $\Lambda \subset \Delta$. The proof is similar to the situation of regular (local) Gibbs specifications [12, Proposition 2.5].

Let $\mathcal{C}_{b}\left(\Omega^{\nabla}\right)$ be the set of bounded functions on $\Omega^{\nabla}$. Gradient Gibbs measures will now be defined in the usual way by having their conditional probabilities outside finite regions prescribed by the gradient Gibbs specification:

Definition 3.2. A measure $\nu \in \mathcal{M}_{1}\left(\Omega^{\nabla}\right)$ is called a gradient Gibbs measure (GGM) if it satisfies the DLR equation

$$
\int \nu(d \zeta) F(\zeta)=\int \nu(d \zeta) \int \gamma_{\Lambda}^{\prime}(d \tilde{\zeta} \mid \zeta) F(\tilde{\zeta})
$$

for every finite $\Lambda \subset V$ and for all $F \in \mathcal{C}_{b}\left(\Omega^{\nabla}\right)$. The set of gradient Gibbs measures will be denoted by $\mathcal{G}^{\nabla}(\gamma)$. 
Construction of GGMs via boundary laws:

In what follows we may assume the a-priori measure $\nu$ on $\mathbb{Z}$ to be the counting measure. On trees with nearest-neighbours potentials $\Phi$ such as the one we consider here, it is possible to use the natural orientations of edges to introduce tree-indexed Markov chains. These are probability measures $\mu$ having the property that for all oriented edges $\langle x y\rangle$ and any $\omega_{y} \in E$,

$$
\mu\left(\sigma_{y}=\omega_{y} \mid \mathcal{F}_{(-\infty, x y)}\right)=\mu\left(\sigma_{y}=\omega_{y} \mid \mathcal{F}_{x}\right) \quad \mu \text {-a.s., }
$$

where

$$
(-\infty, x y):=\left\{w \in V \mid\langle x, y\rangle \in \vec{L}_{w}\right\},
$$

denotes the past of the edge $\langle x, y\rangle$. One can associate to $\mu$ a transition matrix defined to be any stochastic matrix $P=\left(P_{x y}\right)_{\langle x y\rangle}$ satisfying for all $\omega_{y} \in E$

$$
\mu\left(\sigma_{y}=\omega_{y} \mid \mathcal{F}_{x}\right)=P_{x y}\left(\sigma_{x}, \omega_{y}\right) \mu-\text { a.s. }
$$

For n.n. interaction potential $\Phi=\left(\Phi_{b}\right)_{b}$, where bonds are denoted $b=\langle x, y\rangle$, one first defines symmetric transfer matrices $Q_{b}$ following the terminology of Cox [9] or Zachary $[25,26]$ (see also [12]). Setting

$$
Q_{b}\left(\omega_{b}\right)=e^{-\left(\Phi_{b}\left(\omega_{b}\right)+|\partial x|^{-1} \Phi_{\{x\}}\left(\omega_{x}\right)+|\partial y|^{-1} \Phi_{\{y\}}\left(\omega_{y}\right)\right)}
$$

one can rewrite the Gibbsian specification as

$$
\gamma_{\Lambda}^{\Phi}\left(\sigma_{\Lambda}=\omega_{\Lambda} \mid \omega\right)=\left(Z_{\Lambda}^{\Phi}\right)(\omega)^{-1} \prod_{b \cap \Lambda \neq \emptyset} Q_{b}\left(\omega_{b}\right)
$$

If for any bond $b=\langle x, y\rangle$ the transfer operator $Q_{b}\left(\omega_{b}\right)$ is a function of gradient spin variable $\zeta_{b}=\omega_{y}-\omega_{x}$ we call the underlying potential $\Phi$ a gradient interaction potential.

Now we note the following: On the one hand, each extreme Gibbs measure on a tree with respect to a Markov specification is a tree-indexed Markov chain (Theorem 12.6 in [12]). On the other hand (Lemma 3.1 in [25]), a measure $\mu$ is a Gibbs measure with respect to a nearest neighbour potential $\Phi$ with associated family of transfer matrices $\left(Q_{b}\right)_{b \in L}$ iff its marginals at any finite volume $\Lambda \subset V$ are of the form

$$
\mu\left(\sigma_{\Lambda \cup \partial \Lambda}=\omega_{\Lambda \cup \partial \Lambda}\right)=c_{\Lambda}\left(\omega_{\partial \Lambda}\right) \prod_{b \cap \Lambda \neq \emptyset} Q_{b}\left(\omega_{b}\right)
$$

for some function $c_{\Lambda}: \partial \Lambda \rightarrow \mathbb{R}_{+}$. Taking this into account leads to the concept of boundary laws that allows to describe the Gibbs measures that are Markov chains on trees.

Definition 3.3. A family of vectors $\left\{l_{x y}\right\}_{\langle x, y\rangle \in \vec{L}}$ with $l_{x y} \in(0, \infty)^{\mathbb{Z}}$ is called a boundary law for the transfer operators $\left\{Q_{b}\right\}_{b \in L}$ if for each $\langle x, y\rangle \in \vec{L}$ there exists a constant $c_{x y}>0$ such that the consistency equation

$$
l_{x y}\left(\omega_{x}\right)=c_{x y} \prod_{z \in \partial x \backslash\{y\}} \sum_{\omega_{z} \in \mathbb{Z}} Q_{z x}\left(\omega_{x}, \omega_{z}\right) l_{z x}\left(\omega_{z}\right)
$$

holds for every $\omega_{x} \in \mathbb{Z}$. A boundary law is called to be $q$-periodic if $l_{x y}\left(\omega_{x}+q\right)=l_{x y}\left(\omega_{x}\right)$ for every oriented edge $\langle x, y\rangle \in \vec{L}$ and each $\omega_{x} \in \mathbb{Z}$.

In our unbounded discrete context, there is as in the finite-state space context, a one-to-one correspondence between boundary laws and tree-indexed Markov chains, but for some boundary laws only, the ones that are normalisable in the sense of Zachary [25, 26]. 
Definition 3.4 (Normalisable boundary laws). A boundary law $l$ is said to be normalisable if and only if

$$
\sum_{\omega_{x} \in \mathbb{Z}}\left(\prod_{z \in \partial x} \sum_{\omega_{z} \in \mathbb{Z}} Q_{z x}\left(\omega_{x}, \omega_{z}\right) l_{z x}\left(\omega_{z}\right)\right)<\infty
$$

at any $x \in V$.

The correspondence now reads the following:

Theorem 3.5 (Theorem 3.2 in [25]). For any Markov specification $\gamma$ with associated family of transfer matrices $\left(Q_{b}\right)_{b \in L}$ we have

1. Each normalisable boundary law $\left(l_{x y}\right)_{x, y}$ for $\left(Q_{b}\right)_{b \in L}$ defines a unique tree-indexed Markov chain $\mu \in \mathcal{G}(\gamma)$ via the equation given for any connected set $\Lambda \in \mathcal{S}$

$$
\mu\left(\sigma_{\Lambda \cup \partial \Lambda}=\omega_{\Lambda \cup \partial \Lambda}\right)=\left(Z_{\Lambda}\right)^{-1} \prod_{y \in \partial \Lambda} l_{y y_{\Lambda}}\left(\omega_{y}\right) \prod_{b \cap \Lambda \neq \emptyset} Q_{b}\left(\omega_{b}\right),
$$

where for any $y \in \partial \Lambda, y_{\Lambda}$ denotes the unique n.n. of $y$ in $\Lambda$.

2. Conversely, every tree-indexed Markov chain $\mu \in \mathcal{G}(\gamma)$ admits a representation of the form (3.15) in terms of a normalisable boundary law (unique up to a constant positive factor).

Remark 3.6. The Markov chain $\mu$ defined in (3.15) has the transition probabilities

$$
P_{i j}\left(\omega_{i}, x\right)=\mu\left(\sigma_{j}=x \mid \sigma_{i}=\omega_{i}\right)=\frac{l_{j i}(x) Q_{j i}\left(x, \omega_{i}\right)}{\sum_{y} l_{j i}(y) Q_{j i}\left(y, \omega_{i}\right)} .
$$

The expressions (3.16) may exist even in situations where the underlying boundary law $\left(l_{x y}\right)_{x, y}$ is not normalisable in the sense of Definition 3.4. However, the Markov chain given by the so defined transition probabilities is in general not positively recurrent which means that it does not possess an invariant probability measure. More precisely if the Markov chain defined by (3.16) is of the form (3.15) (and hence of the form (3.12)) then its underlying boundary law must be necessarily normalisable as one can see by considering (3.15) for $\Lambda=\{x\}, x \in V$. Thus, there is no obvious extension of Theorem 3.5 to non-normalisable boundary laws.

Remark 3.7. For $k \geq 2$ we also expect tree-automorphism invariant proper Gibbs measures for the SOS-model (2.1) to exist at sufficiently low temperatures, but are not aware of a result in the literature. To prove this conjecture, one needs to show existence of nonperiodic normalisable solutions to the boundary law equation (3.13).

Let us now assume that $Q_{b}=Q$ for all $b \in L$ (this holds obviously true for the SOS model). We call a vector $l \in(0, \infty)^{\mathbb{Z}}$ a (spatially homogeneous) boundary law if there exists a constant $c>0$ such that the consistency equation

$$
l(i)=c\left(\sum_{j \in \mathbb{Z}} Q(i, j) l(j)\right)^{k}
$$

is satisfied for every $i \in \mathbb{Z}$.

Now assume that the elements of the family $\left(Q_{b}\right)_{b \in L}$ do not depend on the bonds i.e. $Q_{b}=Q$ for all $b \in L$, i.e. the underlying potential is tree-automorphism invariant.

In the case of spatially homogeneous boundary laws the expression (3.14) in the definition of normalisability reads

$$
\begin{aligned}
\sum_{i \in \mathbb{Z}}\left(\sum_{j \in \mathbb{Z}} Q(i, j) l(j)\right)^{k+1} & =\sum_{i \in \mathbb{Z}} c^{-\frac{k+1}{k}}\left(c\left(\sum_{j \in \mathbb{Z}} Q(i, j) l(j)\right)^{k}\right)^{\frac{k+1}{k}} \\
& =c^{-\frac{k+1}{k}} \sum_{i \in \mathbb{Z}} l(i)^{\frac{k+1}{k}}
\end{aligned}
$$


which means that any spatially homogeneous normalisable boundary law is an element of the space $l^{1+\frac{1}{k}}$. Thus height-periodic spatially homogeneous boundary laws are never normalisable in the sense of Definition 3.4.

However, it is possible to assign (tree-automorphism invariant) gradient Gibbs measures to spatially homogeneous height-periodic boundary laws to tree-automorphism invariant gradient interaction potentials. The main idea consists in considering for any boundary law $\left(l_{x y}\right)$ to a gradient interaction potential and any finite connected subset $\Lambda \subset V$ the (in general only $\sigma$-finite) measure $\mu_{\Lambda}$ on $\left(\mathbb{Z}^{\Lambda \cup \partial \Lambda}, \otimes_{i \in \Lambda \cup \partial \Lambda} 2^{\mathbb{Z}}\right)$ given by the assignment (3.15), i.e.

$$
\mu_{\Lambda \cup \partial \Lambda}\left(\sigma_{\Lambda \cup \partial \Lambda}=\omega_{\Lambda \cup \partial \Lambda}\right)=\prod_{y \in \partial \Lambda} l_{y y_{\Lambda}}\left(\omega_{y}\right) \prod_{b \cap \Lambda \neq \emptyset} Q_{b}\left(\omega_{b}\right) .
$$

Then fix any pinning site $w \in \Lambda$ and identify $\mu_{\Lambda}$ with its pushforward measure on $\mathbb{Z}^{\vec{L}} \times \mathbb{Z}$ under (3.3). This measure has the marginals

$$
\begin{aligned}
\mu_{\Lambda \cup \partial \Lambda}\left(\sigma_{w}=i, \eta_{\Lambda \cup \partial \Lambda}=\zeta_{\Lambda \cup \partial \Lambda}\right) & =\mu_{\Lambda \cup \partial \Lambda}\left(\sigma_{w}=i\right) \mu_{\Lambda \cup \partial \Lambda}\left(\eta_{\Lambda \cup \partial \Lambda}=\zeta_{\Lambda \cup \partial \Lambda} \mid \sigma_{w}=i\right) \\
& =\mu_{\Lambda \cup \partial \Lambda}\left(\sigma_{w}=i\right) \prod_{y \in \partial \Lambda} l_{y y_{\Lambda}}\left(i+\sum_{b \in \Gamma(w, y)} \zeta_{b}\right) \prod_{b \cap \Lambda \neq \emptyset} Q_{b}\left(\zeta_{b}\right) .
\end{aligned}
$$

If the boundary law $l$ is assumed to be period- $q$ height-periodic, then $\mu_{\Lambda \cup \partial \Lambda}\left(\eta_{\Lambda \cup \partial \Lambda}=\cdot \mid\right.$ $\sigma_{w}=i$ ) will depend on $i$ only modulo $q$. For any class label $s \in \mathbb{Z}_{q}$ this allows us to obtain a probability measure $\nu_{w, s}$ on $\mathbb{Z}^{\{b \in \vec{L} \mid b \subset \Lambda\}}$ by setting

$$
\begin{aligned}
\nu_{w, s}\left(\eta_{\Lambda \cup \partial \Lambda}=\zeta_{\Lambda \cup \partial \Lambda}\right) & :=Z_{w, s}^{\Lambda} \mu_{\Lambda \cup \partial \Lambda}\left(\eta_{\Lambda \cup \partial \Lambda}=\zeta_{\Lambda \cup \partial \Lambda} \mid \sigma_{w}=s\right) \\
& =Z_{w, s}^{\Lambda} \prod_{y \in \partial \Lambda} l_{y y_{\Lambda}}\left(T_{q}\left(s+\sum_{b \in \Gamma(w, y)} \zeta_{b}\right)\right) \prod_{b \cap \Lambda \neq \emptyset} Q_{b}\left(\zeta_{b}\right),
\end{aligned}
$$

where $Z_{w, s}^{\Lambda}$ is a normalization constant and $T_{q}: \mathbb{Z} \rightarrow \mathbb{Z}_{q}$ denotes the coset projection. Then one can show the following:

Theorem 3.8 (Theorem 3.1 in [15]). Let $\left(l_{\langle x y\rangle}\right)_{<x, y>\in \vec{L}}$ be any period-q height-periodic boundary law to some gradient interaction potential. Fix any site $w \in V$ and any class label $s \in \mathbb{Z}_{q}$. Then the definition

$$
\nu_{w, s}\left(\eta_{\Lambda \cup \partial \Lambda}=\zeta_{\Lambda \cup \partial \Lambda}\right)=Z_{w, s}^{\Lambda} \prod_{y \in \partial \Lambda} l_{y y_{\Lambda}}\left(T_{q}\left(s+\sum_{b \in \Gamma(w, y)} \zeta_{b}\right)\right) \prod_{b \cap \Lambda \neq \emptyset} Q_{b}\left(\zeta_{b}\right),
$$

where $\Lambda$ with $w \in \Lambda \subset V$ is any finite connected set, $\zeta_{\Lambda \cup \partial \Lambda} \in \mathbb{Z}^{\{b \in \vec{L} \mid b \subset(\Lambda \cup \partial \Lambda)\}}$ and $\mathbb{Z}_{w, s}^{\Lambda}$ is a normalization constant, gives a consistent family of probability measures on the gradient space $\Omega^{\nabla}$. The measures $\nu_{w, s}$ will be called pinned gradient measures.

By construction, the pinned gradient measures $\nu_{w, s}$ on $\Omega^{\nabla}$ have a restricted gradient (Gibbs) property in the sense that the DLR-equation (3.11) holds for any finite subgraph $\Lambda \subset V$ which does not contain the pinning site $w$ (for details see [15]). If the period$q$ height-periodic boundary law is now additionally spatially homogeneous and the underlying potential is tree-automorphism invariant then it is possible to obtain a treeautomorphism invariant probability measure $\nu$ on the the gradient space by mixing the pinned gradient measures over an appropriate distribution on $\mathbb{Z}_{q}$. In particular, the so-obtained measure $\nu$ is independent of the choice of the pinning site. Thus, the restricted gradient Gibbs property of each of the pinned gradient measures leads to the Gibbs property of the measure $\nu$. 
Theorem 3.9 (Theorem 4.1, Remark 4.2 in [15]). Let $l$ be any spatially homogeneous period-q height-periodic boundary law to a tree-automorphism invariant gradient interaction potential on the Cayley tree. Let $\Lambda \subset V$ be any finite connected set and let $w \in \Lambda$ be any vertex. Then the measure $\nu$ with marginals given by

$$
\nu\left(\eta_{\Lambda \cup \partial \Lambda}=\zeta_{\Lambda \cup \partial \Lambda}\right)=Z_{\Lambda}\left(\sum_{s \in \mathbb{Z}_{q}} \prod_{y \in \partial \Lambda} l\left(s+\sum_{b \in \Gamma(w, y)} \zeta_{b}\right)\right) \prod_{b \cap \Lambda \neq \emptyset} Q\left(\zeta_{b}\right),
$$

where $Z_{\Lambda}$ is a normalisation constant, defines a spatially homogeneous GGM on $\Omega^{\nabla}$.

Remark 3.10. Setting $n_{i}^{w}\left(\zeta_{\Lambda \cup \partial \Lambda}\right):=\left|\left\{y \in \partial \Lambda \mid \sum_{b \in \Gamma(w, y)} \zeta_{b} \equiv i \bmod q\right\}\right|$ the marginals of the measure $\nu$ defined in Theorem 3.9 can be written in the form:

$$
\begin{aligned}
\nu\left(\eta_{\Lambda \cup \partial \Lambda}=\zeta_{\Lambda \cup \partial \Lambda}\right) & =Z_{\Lambda}\left(\sum_{j \in \mathbb{Z}_{q}} \prod_{i \in \mathbb{Z}_{q}} l_{i}^{n_{i+j}^{w}\left(\zeta_{\Lambda \cup \partial \Lambda}\right)}\right) \prod_{b \cap \Lambda \neq \emptyset} Q\left(\zeta_{b}\right) \\
& =Z_{\Lambda}\left(\sum_{j \in \mathbb{Z}_{q}} \prod_{i \in \mathbb{Z}_{q}} l_{i+j}^{n_{i}^{w}\left(\zeta_{\Lambda \cup \partial \Lambda}\right)}\right) \prod_{b \cap \Lambda \neq \emptyset} Q\left(\zeta_{b}\right) .
\end{aligned}
$$

This representation directly shows that two height-periodic boundary laws will lead to the same GGM if one is obtained from the other by a cyclic permutation or multiplication with a positive constant.

To obtain sufficient criteria for two GGM $\nu^{l}$ and $\nu^{\tilde{l}}$ associated to two distinct heightperiodic boundary laws $l$ and $\tilde{l}$ with $l_{0}=\tilde{l}_{0}=1$ being distinct we first observe that $\nu^{l}=\nu^{\tilde{l}}$ if and only if

$$
\frac{\sum_{j \in \mathbb{Z}_{q}} \prod_{i \in \mathbb{Z}_{q}} l_{i}^{n_{i+j}^{w}\left(\zeta_{\Lambda \cup \partial \Lambda}\right)}}{\sum_{j \in \mathbb{Z}_{q}} \prod_{i \in \mathbb{Z}_{q}} \tilde{l}_{i}^{n_{i+j}^{w}\left(\zeta_{\Lambda \cup \partial \Lambda}\right)}}=\frac{Z_{\Lambda}^{\tilde{l}}}{Z_{\Lambda}^{l}}
$$

for all finite subtrees $\Lambda$ and $\zeta_{\Lambda \cup \partial \Lambda} \in \mathbb{Z}^{\{b \in L \mid b \subset \Lambda \cap \partial \Lambda\}}$.

Thus $\nu^{l}=\nu^{l}$, if and only if for any finite subtree $\Lambda$ there is a constant $c(\Lambda)>0$, such that

$$
\sum_{j \in \mathbb{Z}_{q}} \prod_{i \in \mathbb{Z}_{q}} l_{i}^{n_{i+j}}=c(\Lambda) \sum_{j \in \mathbb{Z}_{q}} \prod_{i \in \mathbb{Z}_{q}} \tilde{l}_{i}^{n_{i+j}}
$$

for all vectors $\left(n_{0}, n_{2}, \ldots, n_{q-1}\right) \in \mathbb{N}_{0}$ with $\sum_{i \in \mathbb{Z}_{q}} n_{i}=|\partial \Lambda|$. If we take a single-bond volume $\Lambda=\{b\}$, where $b \in L$, we obtain the marginal

$$
\nu\left(\eta_{b}=\zeta_{b}\right)=Z_{b} \sum_{s \in \mathbb{Z}_{q}} l(s) l\left(s+\zeta_{b}\right) Q\left(\zeta_{b}\right)=Z_{b}\left(\sum_{j \in \mathbb{Z}_{q}} \prod_{i \in \mathbb{Z}_{q}} l_{i}^{n_{i+j}\left(\zeta_{b}\right)}\right) Q_{b}\left(\zeta_{b}\right) .
$$

From this we get that if $\nu^{l}=\nu^{\tilde{l}}$ then condition (3.25) is fulfilled for all vectors $\left(n_{0}, n_{1}, \ldots, n_{q-1}\right) \in\{0,1,2\}^{q}$ with $\sum_{i \in \mathbb{Z}_{q}} n_{i}=2$.

We will now conclude some statements on identifiability of GGM with respect to the class of boundary laws which we will describe in the following section.

Lemma 3.11. Let $l$ and $\tilde{l}$ be two period-2 height-periodic boundary laws with $l_{0}=\tilde{l}_{0}=1$. Denote $l_{1}=a_{1}, \tilde{l}_{1}=a_{2}$.

Then

$$
\nu^{l}=\nu^{\tilde{l}} \quad \text { if and only if } a_{1}=a_{2} \text { or } a_{1} a_{2}=1
$$

Proof. Let us first prove that $\nu^{l}=\nu^{\tilde{l}}$ if $a_{1}=a_{2}$ or $a_{1} a_{2}=1$. Using the marginals representation given in Remark 3.10 we have that $\nu^{l}=\nu^{\tilde{l}}$ if and only if

$$
\frac{\sum_{j \in\{0,1\}} \prod_{i \in\{0,1\}} l_{i}^{n_{i+j}^{w}\left(\zeta_{\Lambda \cup \partial \Lambda}\right)}}{\sum_{j \in\{0,1\}} \prod_{i \in\{0,1\}} \tilde{l}_{i}^{n_{i+j}^{w}\left(\zeta_{\Lambda \cup \partial \Lambda}\right)}}=\operatorname{const}(\Lambda \cup \partial \Lambda)
$$


for any finite subtree $\Lambda$ and $\zeta_{\Lambda \cup \partial \Lambda} \in \mathbb{Z}^{\{b \in L \mid b \subset \Lambda \cup \partial \Lambda\}}$. Let $n=|\partial \Lambda| \geq 3$ then the vectors $\left(n_{0}, n_{1}\right)\left(\zeta_{\Lambda \cup \partial \Lambda}\right)$ are of the form $(n-m, m)$ for some integer $0 \leq m \leq n$. Inserting this into (3.28) we conclude that $\nu^{l}=\nu^{\tilde{l}}$ if and only if for all $n \geq 3$ which can be realized as the number of points in the boundary of a finite subtree and any $0 \leq m_{1}, m_{2} \leq n$ we have:

$$
\frac{a_{1}^{n-m_{1}}+a_{1}^{m_{1}}}{a_{2}^{n-m_{1}}+a_{2}^{m_{1}}}=\frac{a_{1}^{n-m_{2}}+a_{1}^{m_{1}}}{a_{2}^{n-m_{2}}+a_{2}^{m_{2}}} .
$$

We may further assume $m_{1}=0$ and write $m=m_{2}$. Then this equation reduces to

$$
\frac{a_{1}^{n}+1}{a_{2}^{n}+1}=\frac{a_{1}^{n-m}+a_{1}^{m}}{a_{2}^{n-m}+a_{2}^{m}},
$$

which holds true if $a_{2}=\left(a_{1}\right)^{-1}$.

To prove the other direction we must show that $a_{1}=a_{2}$ and $a_{1} a_{2}=1$ are the only solutions to the system (3.30):

For any $x>0$ set $f_{(n, m)}(x):=\frac{x^{n}+1}{x^{n-m}+x^{m}}$. Then (3.30) is equivalent to $f_{(n, m)}\left(a_{1}\right)=$ $f_{(n, m)}\left(a_{2}\right)$. Consider any $0<m<n$. Clearly $f_{(n, m)}$ is continuous, strictly decreasing on $(0,1)$ and strictly increasing on $(1, \infty)$, which means that for any $x \in(0,1]$ there is at most one $y \in[1, \infty)$ with $f_{(n, m)}(x)=f_{(n, m)}(y)$. Since $f_{(n, m)}(x)=f_{(n, m)}\left(\frac{1}{x}\right)$ we have that $f_{(n, m)}\left(a_{1}\right)=f_{(n, m)}\left(a_{2}\right)$ if and only if $a_{1}=a_{2}$ or $a_{1} a_{2}=0$.

Lemma 3.12. Consider any period-4 height-periodic boundary law of the type

$$
l_{i}^{(a, b)}=\left\{\begin{array}{l}
1, \text { if } i \equiv 0 \quad \text { or } 2 \quad \bmod 4 \\
a, \text { if } i \equiv 1 \quad \bmod 4 \\
b, \text { if } i \equiv 3 \quad \bmod 4
\end{array}\right.
$$

and denote the associated GGM by $\nu^{(a, b)}$. Let $\left(a_{1}, b_{1}\right),\left(a_{2}, b_{2}\right)$ be two such boundary laws. If $\nu^{\left(a_{1}, b_{1}\right)}=\nu^{\left(a_{2}, b_{2}\right)}$ then necessarily

$$
\begin{aligned}
& a_{1}+b_{1}=a_{2}+b_{2} \text { or } \\
& \left(a_{i}+b_{i}\right)\left(a_{j}+b_{j}\right)=4 .
\end{aligned}
$$

Proof. Consider the marginal on a set $\Lambda:=\{b\}$, where $b \in L$ is any edge. Inserting the vectors $\left(n_{0}, n_{1}, n_{2}, n_{3}\right)=(2,0,0,0),(1,1,0,0)$ and $(1,0,1,0)$ into (3.25) we conclude that if $\nu^{\left(a_{1}, b_{1}\right)}=\nu^{\left(a_{2}, b_{2}\right)}$ then there is some constant $c>0$ with

1. $a_{1}^{2}+b_{1}^{2}+2=c\left(a_{2}^{2}+b_{2}^{2}+2\right)$,

2. $a_{1}+b_{1}=c\left(a_{2}+b_{2}\right)$ and

3. $1+a_{1} b_{1}=c\left(1+a_{2} b_{2}\right)$.

Adding twice the third equation to the first we obtain

$$
\left(a_{1}+b_{1}\right)^{2}+4=c\left(\left(a_{2}+b_{2}\right)^{2}+4\right),
$$

which in combination with (2) gives

$$
\frac{\left(a_{1}+b_{1}\right)^{2}+4}{\left(a_{2}+b_{2}\right)^{2}+4}=\frac{a_{1}+b_{1}}{a_{2}+b_{2}} .
$$

Setting $x:=a_{1}+b_{1}$ and $y:=a_{2}+b_{2}$ leads to the equation $\frac{x^{2}+4}{y^{2}+4}=\frac{x}{y}$ which is equivalent to

$$
(x-y)(x y-4)=0 .
$$

This completes the proof. 
Lemma 3.13. Consider the $k$-regular tree, $k \geq 2$, and a period-3 height-periodic boundary law of the type

$$
l_{i}^{(c)}= \begin{cases}1, & \text { if } i \equiv 0 \quad \bmod 3 \\ c, & \text { else }\end{cases}
$$

Denote the associated GGM by $\nu^{(c)}$. Then the following holds true:

a) $\nu^{\left(c_{1}\right)}=\nu^{\left(c_{2}\right)}$ if and only if for any $n \in \mathbb{N}$ we have $f_{\left(m_{0}, m_{1}, m_{2}\right)}\left(c_{1}\right)=f_{\left(m_{0}, m_{1}, m_{2}\right)}\left(c_{2}\right)$ for all $\left.\left(m_{0}, m_{1}, m_{2}\right) \in\{0,1, \ldots, n(k-1)+2)\right\}^{3}$ with $m_{0}+m_{1}+m_{2}=2(n(k-1)+2)$, where

$$
f_{\left(m_{0}, m_{1}, m_{2}\right)}(x):=\frac{x^{m_{0}}+x^{m_{1}}+x^{m_{2}}}{1+2 x^{n(k-1)+2}}, \quad x>0 .
$$

b) $\nu^{\left(c_{1}\right)}=\nu^{\left(c_{2}\right)}$ if and only if $c_{1}=c_{2}$.

c) The GGMs associated to the nontrivial members of this family of solutions are all different from the GGMs associated to the solutions given by the family of boundary laws defined in Lemma 3.12.

Proof. The structure of the proof is similar to the proof of Lemma 3.12:

a) First note that for any subtree of the $k$-regular tree with $n$ vertices we have $n(k+1)-2(n-1)=n(k-1)+2$ points in the outer boundary which follows by induction on $n$ (see [22]). Thus $\nu^{\left(c_{1}\right)}=\nu^{\left(c_{2}\right)}$ if and only if for each $n \in \mathbb{N}$ the equation (3.25) holds true for all $\left(n_{0}, n_{1}, n_{2}\right) \in \mathbb{N}_{0}^{3}$ with $n_{0}+n_{1}+n_{2}=n(k-1)+2$. This is equivalent to the existence of some $\lambda>0$ depending only on $k$ and $n$ with

$$
c_{1}^{\left(n_{0}+n_{1}\right)}+c_{1}^{\left(n_{0}+n_{2}\right)}+c_{1}^{\left(n_{1}+n_{2}\right)}=\lambda\left(c_{2}^{\left(n_{0}+n_{1}\right)}+c_{2}^{\left(n_{0}+n_{2}\right)}+c_{2}^{\left(n_{1}+n_{2}\right)}\right),
$$

for all such vectors $\left(n_{0}, n_{1}, n_{2}\right)$.

Setting $\left(\begin{array}{l}m_{0} \\ m_{1} \\ m_{2}\end{array}\right):=\left(\begin{array}{lll}1 & 1 & 0 \\ 1 & 0 & 1 \\ 0 & 1 & 1\end{array}\right)\left(\begin{array}{l}n_{0} \\ n_{1} \\ n_{2}\end{array}\right)$, i.e. $\left(\begin{array}{l}n_{0} \\ n_{1} \\ n_{2}\end{array}\right)=\frac{1}{2}\left(\begin{array}{ccc}1 & 1 & -1 \\ 1 & -1 & 1 \\ -1 & 1 & 1\end{array}\right)\left(\begin{array}{l}m_{0} \\ m_{1} \\ m_{2}\end{array}\right)$ this is equivalent to

$$
c_{1}^{m_{0}}+c_{1}^{m_{1}}+c_{1}^{m_{2}}=\lambda\left(c_{2}^{m_{0}}+c_{2}^{m_{1}}+c_{2}^{m_{2}}\right)
$$

for all $\left(m_{0}, m_{1}, m_{2}\right) \in\{0,1, \ldots, n(k-1)+2\}^{3}$ with $m_{0}+m_{1}+m_{2}=2(n(k-1)+2)$. Hence we have $\nu^{\left(c_{1}\right)}=\nu^{\left(c_{2}\right)}$ if and only if

$$
\frac{c_{1}^{m_{0}}+c_{1}^{m_{1}}+c_{1}^{m_{2}}}{c_{2}^{m_{0}}+c_{2}^{m_{1}}+c_{2}^{m_{2}}}=\frac{c_{1}^{\tilde{m}_{0}}+c_{1}^{\tilde{m}_{1}}+c_{1}^{\tilde{m}_{2}}}{c_{2}^{\tilde{m}_{0}}+c_{2}^{\tilde{m}_{1}}+c_{2}^{\tilde{m}_{2}}}
$$

for all vectors $\left(m_{0}, m_{1}, m_{2}\right),\left(\tilde{m}_{0}, \tilde{m}_{1}, \tilde{m}_{2}\right)$. Fixing $\left(\tilde{m}_{0}, \tilde{m}_{1}, \tilde{m}_{2}\right)=(0, n(k-1)+2, n(k-$ $1)+2$ ) this is equivalent to

$$
\frac{c_{1}^{m_{0}}+c_{1}^{m_{1}}+c_{1}^{m_{2}}}{c_{2}^{m_{0}}+c_{2}^{m_{1}}+c_{2}^{m_{2}}}=\frac{1+2 c_{1}^{n(k-1)+2}}{1+2 c_{2}^{n(k-1)+2}}
$$

for all $\left(m_{0}, m_{1}, m_{2}\right) \in\{0,1, \ldots, n(k-1)+2\}^{3}$ with $m_{0}+m_{1}+m_{2}=2(n(k-1)+2)$ which proves the first statement.

b) Consider a single-bond marginal $\Lambda=\{b\}, b \in L$ and insert the vectors $\left(n_{0}, n_{1}, n_{2}\right)=$ $(2,0,0)$ and $(1,1,0)$ in (3.26). If $\nu^{\left(c_{1}\right)}=\nu^{\left(c_{2}\right)}$ then there is a constant $\lambda>0$ with
i) $1+2 c_{1}^{2}=\lambda\left(1+2 c_{2}^{2}\right)$ and
ii) $c_{1}^{2}+2 c_{1}=\lambda\left(c_{2}^{2}+2 c_{2}\right)$. 
From this we obtain the polynomial equation in $c_{1}$ :

$$
c_{1}^{2}\left(4 c_{2}-1\right)-2 c_{1}\left(1+2 c_{2}^{2}\right)+c_{2}\left(c_{2}+2\right)=0 .
$$

Dividing out the linear term $\left(c_{1}-c_{2}\right)$ we arrive at

$$
c_{1}\left(4 c_{2}-1\right)-c_{2}-2=0 \text {. }
$$

In the second step we will show that the assumption $\nu^{\left(c_{1}\right)}=\nu^{\left(c_{2}\right)}$ and $c_{1} \neq c_{2}$ leads to a contradiction. This will be done by considering 3.13 for $n \rightarrow \infty$. Take any real numbers $0<a_{0}<a_{1}<a_{2}<k-1$ where $a_{0}+a_{1}+a_{2}=2(k-1)$. Then there is a sequence $\left(m_{0}(n), m_{1}(n), m_{2}(n)\right)_{n \in \mathbb{N}}$ such that for all $n \in \mathbb{N}$ we have $\left.\left(m_{0}(n), m_{1}(n), m_{2}(n)\right) \in\{0,1, \ldots, n(k-1)+2)\right\}^{3}$ and $m_{0}(n)+m_{1}(n)+m_{2}(n)=$ $2(n(k-1)+2)$ with the property that $\frac{m_{i}(n)}{n} \stackrel{n \rightarrow \infty}{\rightarrow} a_{i}, i \in\{0,1,2\}$. If $\nu^{\left(c_{1}\right)}=\nu^{\left(c_{2}\right)}$ and $c_{1} \neq c_{2}$ then by (3.33) we have $c_{2}=\frac{c_{1}+2}{4 c_{1}-1}$, so we may assume $\frac{1}{4}<c_{1}<1<c_{2}$. From 3.13 we obtain

$$
\lim _{n \rightarrow \infty} \frac{1}{n} \log f_{\left(m_{0}(n), m_{1}(n), m_{2}(n)\right)}\left(c_{1}\right)=\lim _{n \rightarrow \infty} \frac{1}{n} \log f_{\left(m_{0}(n), m_{1}(n), m_{2}(n)\right)}\left(c_{2}\right)
$$

Hence, taking into account the assumption $0<c_{1}<1<c_{2}$, this implies

$$
a_{0} \log \left(c_{1}\right)=\left(a_{2}-(k-1)\right) \log \left(c_{2}\right),
$$

where the limiting behaviour of the l.h.s can be seen by writing $c_{1}^{m_{0}(n)}+c_{1}^{m_{1}(n)}+$ $c_{1}^{m_{2}(n)}=c_{1}^{m_{0}(n)}\left(1+c_{1}^{m_{1}(n)-m_{0}(n)}+c_{1}^{m_{2}(n)-m_{0}(n)}\right)$ and then inserting $m_{i}(n)=a_{i} n+\varepsilon_{i, n}$ where $\varepsilon_{i, n} \stackrel{n \rightarrow \infty}{\rightarrow} 0$. The r.h.s. follows similarly. Now (3.34) is equivalent to

$$
\frac{\log \left(c_{2}\right)}{\log \left(c_{1}\right)}=\frac{a_{0}}{a_{2}-(k-1)} .
$$

As $c_{2}$ is uniquely given by (3.33) and (3.35) holds true for all choices of $\left(a_{0}, a_{1}, a_{2}\right)$ in the allowed range, the assumption $\nu^{\left(c_{1}\right)}=\nu^{\left(c_{2}\right)}$ and $c_{1} \neq c_{2}$ leads to a contradiction.

c) Let $l^{(a, b)}$ denote any period-4 height-periodic boundary law as defined in Lemma 3.12 and let $l^{(c)}$ be any period-3 height-periodic boundary law as defined above. We will consider each of them as a period-12 height-periodic boundary law. Take $\Lambda=\{b\}, b \in L$ and insert the vectors $\left(n_{0}, n_{1}, \ldots, n_{11}\right)=(1,1,0,0, \ldots, 0)$ and $(1,0,0,1,0,0, \ldots, 0)$ into (3.26). If $\nu^{(a, b)}=\nu^{(c)}$ then there is a constant $\lambda>0$ with
i) $6(a+b)=4 \lambda\left(2 c+c^{2}\right)$ and
ii) $6(a+b)=4 \lambda\left(1+2 c^{2}\right)$

From this we get $c^{2}-2 c+1=0$ which leads to $c=1$.

Remark 3.14. Lemma 3.11 can also be concluded from Lemma 3.12 and the fact that two height-periodic boundary laws lead to the same GGM if one is obtained from the other by cyclic permutations or multiplication with a positive constant.

\section{Tree-automorphism invariant solutions}

In this section we calculate height-periodic solutions to the spatially homogeneous boundary law equation for the SOS-model. 
Remark 4.1. In [24] Shlosman considers the situation on the lattice $\mathbb{Z}^{2}$ with local state space $\mathbb{Z}$ and a general strictly convex symmetric gradient interaction potential (written in the DLR-formalism the results can be found in section 6.3 of [12]). He shows that at sufficiently large $\beta$ there are states which arise as perturbations of staircase-like infinitevolume groundstates. Shlosman's staircase states do not possess reflection-invariance on the lattice by construction, and have a non-zero expectation of the height-difference in the direction of the staircase. By contrast, the gradient states on trees we construct in our paper have the full tree-automorphism invariance. Therefore the expectations of height-differences are zero in our gradient states, in this sense they have "tilt zero".

First let $\beta>0$ be any inverse temperature and set $\theta:=\exp (-\beta)<1$. The transfer operator $Q$ then reads $Q(i-j)=\theta^{|i-j|}$ for any $i, j \in \mathbb{Z}$, and a spatially homogeneous boundary law, now denoted by $z$, is any positive function on $\mathbb{Z}$ solving the system (3.17), whose values we will denote by $z_{i}$ instead of $z(i)$. Further notice that a boundary law is only unique up to multiplication with any positive prefactor. Hence we may choose this constant in a way such that we have $z_{0}=1$. At last set $\mathbb{Z}_{0}:=\mathbb{Z} \backslash\{0\}$. Taking into account these prerequisites, the boundary law equation (3.17) now reads

$$
z_{i}=\left(\frac{\theta^{|i|}+\sum_{j \in \mathbb{Z}_{0}} \theta^{|i-j|} z_{j}}{1+\sum_{j \in \mathbb{Z}_{0}} \theta^{|j|} z_{j}}\right)^{k}, i \in \mathbb{Z}_{0}
$$

\subsection{A simplification of the system (4.1)}

Let $\mathbf{z}(\theta)=\left(z_{i}=z_{i}(\theta), i \in \mathbb{Z}_{0}\right)$ be a solution to (4.1). Denote

$$
l_{i} \equiv l_{i}(\theta)=\sum_{j=-\infty}^{-1} \theta^{|i-j|} z_{j}, \quad r_{i} \equiv r_{i}(\theta)=\sum_{j=1}^{\infty} \theta^{|i-j|} z_{j}, \quad i \in \mathbb{Z}_{0} .
$$

It is clear that each $l_{i}$ and $r_{i}$ can be a finite positive number or $+\infty$.

Lemma 4.2. For each $i \in \mathbb{Z}_{0}$ we have

- $l_{i}<+\infty$ if and only if $l_{0}<+\infty$;

- $r_{i}<+\infty$ if and only if $r_{0}<+\infty$.

Proof. The proof follows from the following equalities

$$
\begin{aligned}
& l_{i}=\left\{\begin{array}{l}
\theta^{i} l_{0}+\sum_{j=i}^{-1}\left(\theta^{j-i}-\theta^{i-j}\right) z_{j}, \quad \text { if } i \leq-1 \\
\theta^{i} l_{0}, \quad \text { if } i \geq 1 .
\end{array}\right. \\
& r_{i}= \begin{cases}\theta^{-i} r_{0}+\sum_{j=1}^{i}\left(\theta^{i-j}-\theta^{j-i}\right) z_{j}, \quad \text { if } i \geq 1 \\
\theta^{-i} r_{0}, \quad \text { if } i \leq-1 .\end{cases}
\end{aligned}
$$

In what follows, we will always assume that $l_{0}<+\infty$ and $r_{0}<+\infty$. Denoting $u_{i}=u_{0} \sqrt[k]{z_{i}}$ (for some $u_{0}>0$ ) from (4.1) we get

$$
u_{i}=C \cdot\left(\cdots+\theta^{2} u_{i-2}^{k}+\theta u_{i-1}^{k}+u_{i}^{k}+\theta u_{i+1}^{k}+\theta^{2} u_{i+2}^{k}+\ldots\right), \quad i \in \mathbb{Z},
$$

for some $C>0$.

This system can be written as

$$
u_{i}=C\left(\sum_{j=1}^{+\infty} \theta^{j} u_{i-j}^{k}+u_{i}^{k}+\sum_{j=1}^{+\infty} \theta^{j} u_{i+j}^{k}\right), \quad i \in \mathbb{Z}
$$


Proposition 4.3. A vector $\mathbf{u}=\left(u_{i}, i \in \mathbb{Z}\right)$, with $u_{0}=1$, is a solution to (4.5) if and only if for $u_{i}\left(=\sqrt[k]{z_{i}}\right)$ the following holds

$$
u_{i}^{k}=\frac{u_{i-1}+u_{i+1}-\tau u_{i}}{u_{-1}+u_{1}-\tau}, \quad i \in \mathbb{Z},
$$

where $\tau=\theta^{-1}+\theta=2 \cosh (\beta)$.

Proof

a) Necessity: From (4.5) we get

$$
\begin{aligned}
& u_{i-1}+u_{i+1} \\
& =C\left(\sum_{j=1}^{\infty} \theta^{j} u_{i-1-j}^{k}+\sum_{j=1}^{\infty} \theta^{j} u_{i+1-j}^{k}+u_{i-1}^{k}+u_{i+1}^{k}+\sum_{j=1}^{\infty} \theta^{j} u_{i-1+j}^{k}+\sum_{j=1}^{\infty} \theta^{j} u_{i+1+j}^{k}\right) \\
& =C\left(\theta^{-1} \sum_{\substack{m=1 \\
m=j+1}}^{\infty} \theta^{m} u_{i-m}^{k}-u_{i-1}^{k}+\theta \sum_{\substack{j^{\prime}=1 \\
j^{\prime}=j-1}}^{\infty} \theta^{j^{\prime}} u_{i-j^{\prime}}^{k}+\theta u_{i}^{k}+u_{i-1}^{k}+u_{i+1}^{k}\right. \\
& \left.+\theta \sum_{n=1}^{\infty} \theta^{n} u_{i+n}^{k}+\theta u_{i}^{k}+\theta^{-1} \sum_{\substack{\bar{j}=1 \\
\bar{j}=j+1}}^{\infty} \theta^{\bar{j}} u_{i+\bar{j}}^{k}-u_{i+1}^{k}\right) \\
& =C\left(\left(\theta^{-1}+\theta\right) \sum_{j=1}^{\infty} \theta^{j} u_{i-j}^{k}+2 \theta u_{i}^{k}+\left(\theta^{-1}+\theta\right) \sum_{j=1}^{\infty} \theta^{j} u_{i+j}^{k}\right) \\
& =\left(\theta^{-1}+\theta\right) u_{i}+C\left(\theta-\theta^{-1}\right) u_{i}^{k} .
\end{aligned}
$$

Thus

$$
u_{i-1}+u_{i+1}-\left(\theta^{-1}+\theta\right) u_{i}=C\left(\theta-\theta^{-1}\right) u_{i}^{k}, \quad i \in \mathbb{Z} .
$$

Since $u_{0}=1$ dividing both sides of (4.7) by the expression obtained in the case $i=0$ we get (4.6).

b) Sufficiency: Assume (4.6) holds. Then we get (4.7) with some $C=\tilde{C}$. Write this equality for $i$ replaced by $i+1-j$, i.e.

$$
u_{i-j}+u_{i-j+2}-\left(\theta^{-1}+\theta\right) u_{i+1-j}=\tilde{C}\left(\theta-\theta^{-1}\right) u_{i+1-j}^{k}, \quad i, j \in \mathbb{Z} .
$$

Multiply both sides of (4.8) by $\theta^{j}$ and sum over $j=1,2, \ldots$ Here, absolute convergence of all occurring infinite sums is guaranteed by the assumption $l_{0}<+\infty$ and $r_{0}<+\infty$ and the fact that $\theta<1$. Then after rearrangement/simplifications we get

$$
\theta u_{i+1}-u_{i}=\tilde{C}\left(\theta-\theta^{-1}\right) \sum_{j=1}^{\infty} \theta^{j} u_{i+1-j}^{k}
$$

Dividing both sides of this equality by $\theta$ we get

$$
u_{i+1}-\theta^{-1} u_{i}=\tilde{C}\left(\theta-\theta^{-1}\right) \sum_{j=1}^{\infty} \theta^{j-1} u_{i+1-j}^{k}=\tilde{C}\left(\theta-\theta^{-1}\right)\left(\sum_{\substack{m=1 \\ m=j-1}}^{\infty} \theta^{m} u_{i-m}^{k}+u_{i}^{k}\right) \text {. }
$$

Now rewrite (4.7) for $i$ replaced by $i+j, C$ is replaced by $\tilde{C}$ and multiply both sides of the obtained equality by $\theta^{j}$ then sum over $j=1,2, \ldots$ After simplifications we 
get

$$
\theta u_{i}-u_{i+1}=\tilde{C}\left(\theta-\theta^{-1}\right) \sum_{j=1}^{\infty} \theta^{j} u_{i+j}^{k} .
$$

Adding (4.9) and (4.10) we get the $i$ th equation of (4.5) with $C$ replaced by $\tilde{C}$. For $u_{0}=1$ we get $\tilde{C}=C$.

Lemma 4.4. If $l_{0}<+\infty$ and $r_{0}<+\infty$ then we have

$$
l_{0}=\frac{\theta-u_{-1}}{u_{-1}+u_{1}-\tau}, \quad r_{0}=\frac{\theta-u_{1}}{u_{-1}+u_{1}-\tau} .
$$

Proof. Using (4.6) we get

$$
l_{0}=\sum_{j=-\infty}^{-1} \theta^{-j} z_{j}=\sum_{j=-\infty}^{-1} \theta^{-j} u_{j}^{k}=\sum_{j=-\infty}^{-1} \theta^{-j} \frac{u_{j-1}+u_{j+1}-\tau u_{j}}{u_{-1}+u_{1}-\tau} .
$$

Compute the following

$$
\begin{aligned}
& \sum_{j=-\infty}^{-1} \theta^{-j}\left(u_{j-1}+u_{j+1}-\tau u_{j}\right) \\
& =\theta^{-1} \sum_{j=-\infty}^{-1} \theta^{-j+1} u_{j-1}+\theta \sum_{j=-\infty}^{-1} \theta^{-j-1} u_{j+1}-\tau \sum_{j=-\infty}^{-1} \theta^{-j} u_{j} \\
& =\theta-u_{-1}+\left(\theta^{-1}+\theta-\tau\right) \sum_{j=-\infty}^{-1} \theta^{-j} u_{j} .
\end{aligned}
$$

Since $\theta^{-1}+\theta-\tau=0$ we get the formula of $l_{0}$. The case $r_{0}$ is similar.

By this Lemma we have

$$
1+l_{0}+r_{0}=\frac{\theta-\theta^{-1}}{u_{-1}+u_{1}-\tau} .
$$

The equation (4.6) can be separated into the following independent recurrence equations

$$
\begin{gathered}
u_{-i-1}=\left(u_{-1}+u_{1}-\tau\right) u_{-i}^{k}+\tau u_{-i}-u_{-i+1}, \\
u_{i+1}=\left(u_{-1}+u_{1}-\tau\right) u_{i}^{k}+\tau u_{i}-u_{i-1},
\end{gathered}
$$

where $i \geq 0, u_{0}=1$ and $u_{-1}, u_{1}$ are some initial numbers. Note that for $i=0$ the above equations are trivially fulfilled for all values of $u_{1}$ and $u_{-1}$. Hence it suffices to consider (4.12) and (4.13) for $i \geq 1$.

\subsection{A class of period- 4 height-periodic solutions to (4.6)}

In this subsection we shall describe the two-parameter family of solutions to (4.6) which have the form

$$
u_{n}=\left\{\begin{array}{l}
1, \text { if } n=0 \text { or } 2 \bmod 4, \\
a, \text { if } n=1 \bmod 4 \\
b, \text { if } n=3 \bmod 4,
\end{array}\right.
$$

where $a$ and $b$ some positive numbers. Such a solution defines a periodic two-sided infinite sequence, i.e.

$$
\ldots, a, 1, b, 1, a, 1, b, 1, a, 1, b, \ldots
$$


The equations (4.12) and (4.13) give the following system of equations

$$
\begin{aligned}
& (a+b-\tau) b^{k}+\tau b-2=0 \\
& (a+b-\tau) a^{k}+\tau a-2=0 .
\end{aligned}
$$

For simplicity we consider the case $k=2$ and give full analysis of the system (4.16).

In case $k=2$ subtracting from the first equation of the system the second one we get

$$
(b-a)\left[(a+b)^{2}-\tau(a+b)+\tau\right]=0 .
$$

This gives three possible cases:

$$
a=b, \text { and } a=\frac{1}{2}\left(\tau \pm \sqrt{\tau^{2}-4 \tau}\right)-b \text { for } \tau \geq 4 .
$$

- Case $a=b$. In this case from the first equation of (4.16) we get

$$
2 a^{3}-\tau a^{2}+\tau a-2=0 .
$$

One easily gets the following solutions to this equation (recall that $\tau>2$ ):

- If $\tau \leq 6$ then the equation (4.18) has unique solution $a_{0}=1$.

- If $\tau>6$ then there are three solutions (see Fig. 1)

$$
a_{0}=1, \quad a_{1}=\frac{1}{4}\left(\tau-2-\sqrt{(\tau-2)^{2}-16}\right), \quad a_{2}=\frac{1}{4}\left(\tau-2+\sqrt{(\tau-2)^{2}-16}\right) .
$$

Note that these 2-periodic solutions can be already found in [15] (recall that $\tau=2 \cosh (\beta))$.

- Case $a+b=\frac{1}{2}\left(\tau+\sqrt{\tau^{2}-4 \tau}\right)$. In this case from the second equation of (4.16) we get

$$
\left(\tau-\sqrt{\tau^{2}-4 \tau}\right) a^{2}-2 \tau a+4=0 .
$$

Which for $\tau \geq 4$ has the solutions

$$
a_{3}=\frac{\tau-\sqrt{\tau^{2}-4 \tau+4 \sqrt{\tau^{2}-4 \tau}}}{\tau-\sqrt{\tau^{2}-4 \tau}}, a_{4}=\frac{\tau+\sqrt{\tau^{2}-4 \tau+4 \sqrt{\tau^{2}-4 \tau}}}{\tau-\sqrt{\tau^{2}-4 \tau}} .
$$

Using (4.17) we get $b_{3}=a_{4}$ and $b_{4}=a_{3}$.

- Case $a+b=\frac{1}{2}\left(\tau-\sqrt{\tau^{2}-4 \tau}\right)$. In this case similarly as in previous case we obtain

$$
\left(\tau+\sqrt{\tau^{2}-4 \tau}\right) a^{2}-2 \tau a+4=0
$$

which for $\tau \geq 2+2 \sqrt{5}$ has the following solutions

$$
a_{5}=\frac{\tau-\sqrt{\tau^{2}-4 \tau-4 \sqrt{\tau^{2}-4 \tau}}}{\tau+\sqrt{\tau^{2}-4 \tau}}, \quad a_{6}=\frac{\tau+\sqrt{\tau^{2}-4 \tau-4 \sqrt{\tau^{2}-4 \tau}}}{\tau+\sqrt{\tau^{2}-4 \tau}} .
$$

Using (4.17) we get $b_{5}=a_{6}$ and $b_{6}=a_{5}$. Clearly all of these solutions are positive (see Fig. 2).

Taking into account the freedom of cyclic permutations of boundary laws we thus proved the following: 


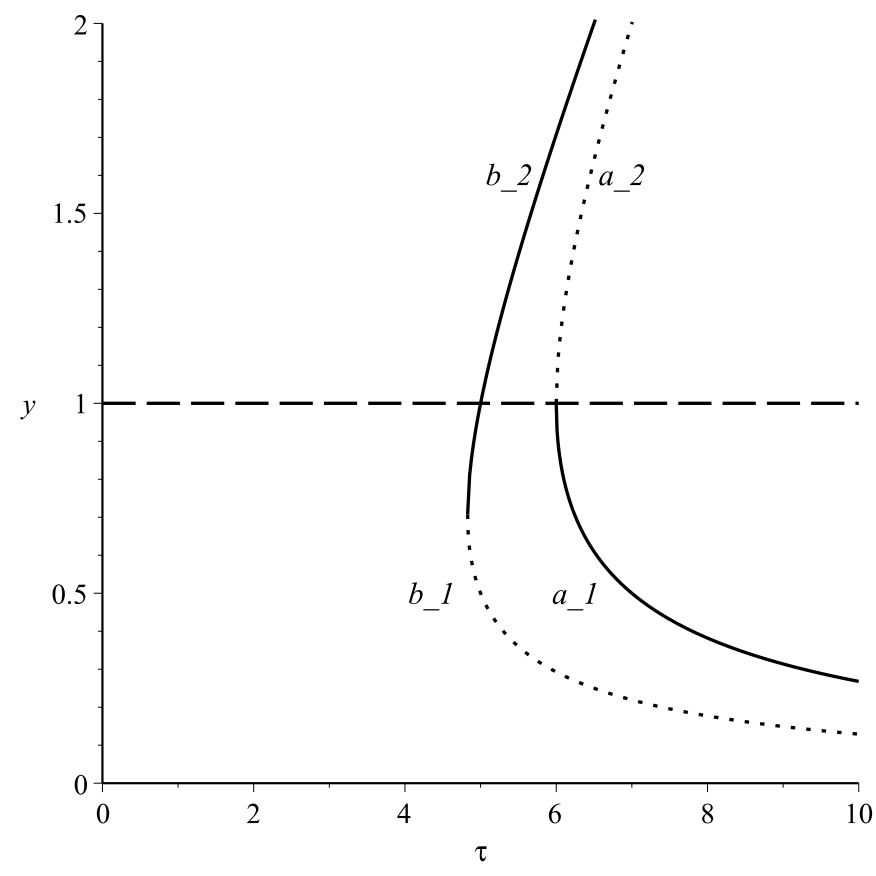

Figure 1: The graphs of the functions $a_{1}, a_{2}$ giving period-2 height-periodic boundary laws. The graphs of the functions $b_{1}, b_{2}$ giving period-3 height-periodic boundary laws. As $a_{2}=a_{1}^{-1}$, both 2-periodic boundary laws lead to the same GGM.

Proposition 4.5. The periodic solutions of the form (4.15) depend on the parameter $\tau=2 \cosh (\beta)$ in the following way.

1. If $\tau \leq 4$ then there is a unique solution with $a=b=1$.

2. If $4<\tau \leq 6$ then there are exactly two solutions with $a=b=1$ and $a=a_{3}, b=b_{3}$.

3. If $6<\tau<2+2 \sqrt{5}$ then there are exactly four solutions with $a=b=1, a=b=a_{1}$, $a=b=a_{2}$ and $(a, b)=\left(a_{3}, b_{3}\right)$.

4. If $\tau \geq 2+2 \sqrt{5}$ then there are exactly five solutions with $a=b=1, a=b=a_{1}$, $a=b=a_{2},(a, b)=\left(a_{3}, b_{3}\right)$ and $(a, b)=\left(a_{5}, b_{5}\right)$,

where the values $a_{i}$ and $b_{i}$ are defined above.

\subsection{Gradient Gibbs measures described by period- 4 height-periodic boundary laws: Identifiability}

In this subsection we will apply the Lemmas 3.11 and 3.12 on identifiability to the gradient Gibbs measures which correspond to the periodic solutions given in Proposition 4.5. Note that a solution described by the parameters $(a, b)$ corresponds to the boundary law

$$
z_{n}^{\left(a^{2}, b^{2}\right)}=\left\{\begin{array}{l}
1, \text { if } n=0 \text { or } 2 \bmod 4, \\
a^{2}, \text { if } n=1 \bmod 4, \\
b^{2}, \text { if } n=3 \bmod 3 .
\end{array}\right.
$$

We will denote the GGM assigned by Theorem 3.9 to a boundary law $z_{n}^{\left(a^{2}, b^{2}\right)}$ by $\nu^{\left(a^{2}, b^{2}\right)}$. 


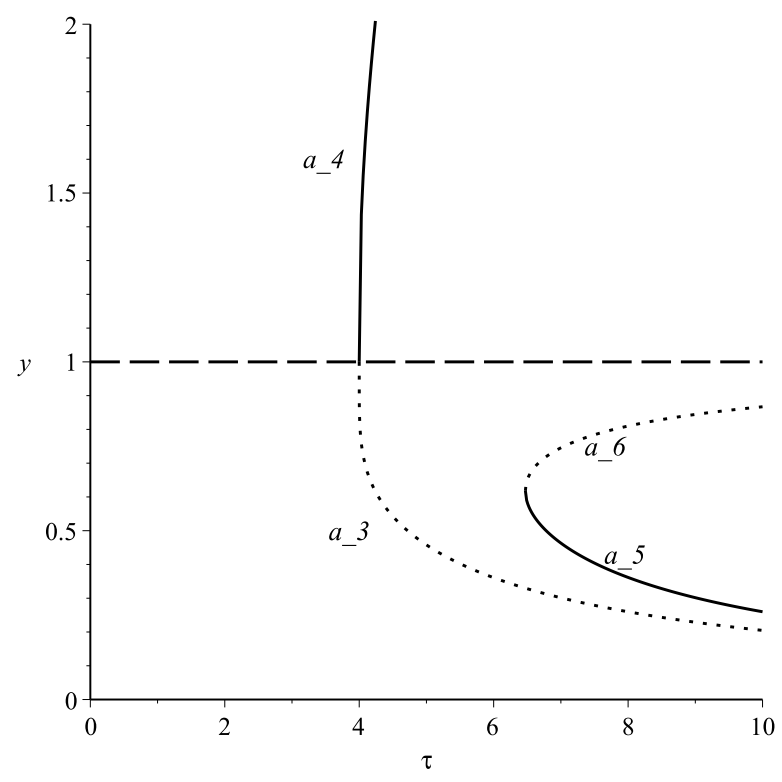

Figure 2: The graphs of the functions $\left(a_{3}, a_{4}\right)$ and $\left(a_{5}, a_{6}\right)$ giving period-4 height-periodic boundary laws.

- Case $4<\tau \leq 6$ : We have $a_{3}^{2}+b_{3}^{2} \geq 2\left(\frac{1}{2}\left(a_{3}+b_{3}\right)\right)^{2}=2\left(\frac{\tau}{\tau-\sqrt{\tau^{2}-4 \tau}}\right)^{2}>2=1^{2}+1^{2}$, so $a_{3}^{2}+b_{3}^{2} \neq 1^{2}+1^{2}$ and $\left(1^{2}+1^{2}\right)\left(a_{3}^{2}+b_{3}^{2}\right) \neq 4$. Thus $\nu^{\left(a_{3}^{2}, b_{3}^{2}\right)} \neq \nu^{(1,1)}$ by Lemma 3.12 .

- Case $6<\tau<2+2 \sqrt{5}$ : We have $a_{1}<1<a_{2}$ and $a_{3}^{2}+b_{3}^{2}>2\left(\frac{1}{16}\left(\tau+\sqrt{\tau^{2}-4 \tau}\right)^{2}\right) \geq 2 a_{2}^{2}$. Further, as $a_{1} a_{2} \equiv 1$, by Lemma 3.11 we have that $\nu^{\left(a_{1}^{2}, a_{1}^{2}\right)} \equiv \nu^{\left(a_{2}^{2}, a_{2}^{2}\right)}$. At last $\left(a_{3}^{2}+b_{3}^{2}\right)\left(a_{2}^{2}+a_{2}^{2}\right)>\left(a_{3}^{2}+b_{3}^{2}\right)\left(a_{1}^{2}+a_{1}^{2}\right)>4\left(a_{1} a_{2}\right)^{2}=4$. Thus we have three different GGMs associated to boundary laws of the type (4.19) via Theorem 3.9.

- Case $\tau \geq 2+2 \sqrt{5}$ : We still have: $a_{1}^{2}<1<a_{2}^{2}<a_{3}^{2}+b_{3}^{2}$ and $a_{1} a_{2} \equiv 1$, so again $\nu^{\left(a_{1}^{2}, a_{1}^{2}\right)} \equiv \nu^{\left(a_{2}^{2}, a_{2}^{2}\right)}$. Further $a_{5}^{2}+b_{5}^{2} \leq 2 b_{5}^{2}<2$. As $a_{1}(\tau)$ is monotonically decreasing in $\tau$ and $a_{5}(\tau)^{2}+b_{5}(\tau)^{2}$ is monotonically increasing in $\tau$ it suffices to numerically calculate $2 a_{1}(2+2 \sqrt{5})^{6}=\left(\frac{1}{2}(\sqrt{5}-1)\right)^{2}<0.4<0.76<a_{5}(2+2 \sqrt{5})^{2}+b_{5}(2+2 \sqrt{5})^{2}$ to obtain: $2 a_{1}^{2}<a_{5}^{2}+b_{5}^{2}<2<2 a_{2}^{2}<a_{3}^{2}+b_{3}^{2}$. Thus we have four different GGMs associated to boundary laws of the type (4.19) via Theorem 3.9.

Hence we have proven the following

Theorem 4.6. For the SOS model (2.1) on the binary tree with parameter $\tau=2 \cosh (\beta)$ the following assertions hold

1. If $\tau \leq 4$ then there is precisely one GGM associated to a boundary law of the type (4.19) via Theorem 3.9.

2. If $4<\tau \leq 6$ then there are precisely two such GGMs.

3. If $6<\tau<2+2 \sqrt{5}$ then there are precisely three such GGMs.

4. If $\tau \geq 2+2 \sqrt{5}$ then there are precisely four such measures. 


\subsection{Period-3 height-periodic boundary laws on the $k$-regular tree.}

To also describe gradient Gibbs measures on the $k$-regular tree for arbitrary $k \geq 2$, we consider a 1-parameter family of period-3 height-periodic boundary laws which can be examined easily. Assume $u_{n}, n \in Z$ has the form

$$
u_{n}=\left\{\begin{array}{lll}
1, & n=0 & \bmod 3 \\
a, & n \neq 0 & \bmod 3
\end{array}\right.
$$

where $a>0$. Then, by (4.13) and (4.12), $a$ should satisfy

$$
2 a^{k+1}-\tau a^{k}+(\tau-1) a-1=0 .
$$

This equation has the solution $a=1$ independently of the parameters $(\tau, k)$. Dividing both sides by $a-1$ we get

$$
2 a^{k}+(2-\tau)\left(a^{k-1}+a^{k-2}+\cdots+a\right)+1=0 .
$$

The equation (4.22) has again the solution $a=1$ iff $\tau=\tau_{0}$, where

$$
\tau_{0}:=\frac{2 k+1}{k-1}
$$

It is well known (see [17], p.28) that the number of positive roots of the polynomial (4.22) does not exceed the number of sign changes of its coefficients. It is obvious that $2-\tau<0$. Thus the number of positive roots of the polynomial (4.22) is at most 2 .

The following lemma gives the full analysis of the equation (4.22):

Lemma 4.7. For each $k \geq 2$, there is exactly one critical value of $\tau=2 \cosh (\beta)$, called $\tau_{c}=\tau_{c}(k)$, such that

1. $\tau_{c}<\tau_{0}$;

2. if $\tau<\tau_{c}$ then (4.22) has no positive solution;

3. if $\tau=\tau_{c}$ then the equation has a unique positive solution;

4. if $\tau>\tau_{c}, \tau \neq \tau_{0}$ then it has exactly two solutions;

5. if $\tau=\tau_{0}$, then the equation has two solutions, one of which is $a=1$.

Proof. Solving (4.22) with respect to $\tau$ we get

$$
\tau=\psi_{k}(a):=2+\frac{2 a^{k}+1}{a^{k-1}+a^{k-2}+\cdots+a} .
$$

We have $\psi_{k}(a)>2, a>0$ and $\psi_{k}^{\prime}(a)=0$ is equivalent to

$$
2 \sum_{j=1}^{k-1}(k-j) a^{k+j-1}-\sum_{j=1}^{k-1} j a^{j-1}=0 .
$$

The last polynomial equation has exactly one positive solution, because signs of its coefficients changed only one time, and at $a=0$ it is negative, i.e. -1 and at $a=+\infty$ it is positive. Denote this unique solution by $a^{*}$. Then $\psi_{k}(a)$ has unique minimum at $a=a^{*}$, and $\lim _{a \rightarrow 0} \psi_{k}(a)=\lim _{a \rightarrow+\infty} \psi_{k}(a)=+\infty$ (see Fig. 3). Thus

$$
\tau_{c}=\tau_{c}(k)=\min _{a>0} \psi_{k}(a)=\psi_{k}\left(a^{*}\right) .
$$

Note that $a^{*} \neq 1$, i.e. $a=1$ does not satisfy (4.23). Therefore

$$
\tau_{0}=\psi_{k}(1)>\tau_{c}=\min _{a>0} \psi_{k}(a)=\psi_{k}\left(a^{*}\right) .
$$

These properties of $\psi_{k}(a)$ completes the proof. 


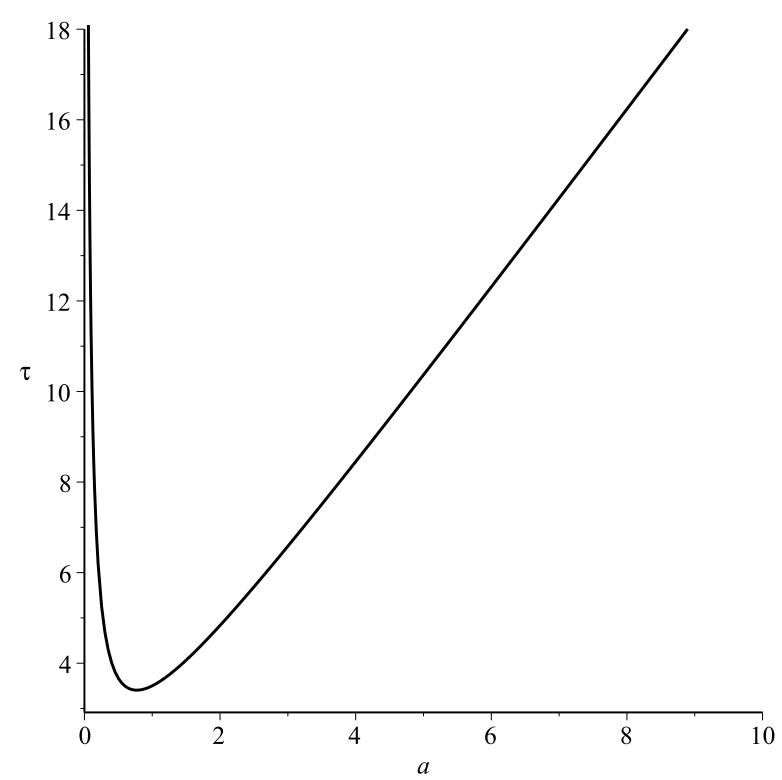

Figure 3: The graphs of the function $\psi_{3}(a)$.

Thus taking into account Lemma 3.13 and Lemma 4.7 we obtain the following:

Theorem 4.8. For the SOS-model on the $k$-regular tree, $k \geq 2$, with parameter $\tau=$ $2 \cosh (\beta)$ there are numbers $0<\tau_{c}<\tau_{0}$ such that the following holds:

1. If $\tau<\tau_{c}$ then there are no GGM corresponding to nontrivial period-3 height-periodic boundary laws of the type (4.20) via Theorem 3.9.

2. At $\tau=\tau_{c}$ there is a unique GGM corresponding to a nontrivial period-3 heightperiodic boundary law of the type (4.20) via Theorem 3.9.

3. For $\tau>\tau_{c}, \tau \neq \tau_{0}$ (resp. $\tau=\tau_{0}$ ) there are exactly two such (resp. one) GGMs.

The GGMs described above are all different from the GGMs mentioned in Theorem 4.6.

Remark 4.9. In case $k=2$ one easily finds $\tau_{c}(2)=2(1+\sqrt{2}) \approx 4.83$ and $\tau_{0}=5$. Two positive solutions are (see Fig. 1):

$$
a=b_{1,2}:=\frac{1}{4}\left(\tau-2 \pm \sqrt{(\tau-2)^{2}-8}\right)
$$

Remark 4.10. It was shown in Section 5 of [15] that the equations for period-3 heightperiodic boundary laws of the general form

$$
z_{i}=\left\{\begin{array}{ccc}
1, & i=0 & \bmod 3 \\
a, & i=1 & \bmod 3 \\
b, & i=2 & \bmod 3
\end{array}\right.
$$

can be identified with the boundary law equations of a Potts model on the same regular tree at a different effective inverse temperature. An explicit discussion of the transition temperature was given only on the binary tree. This correspondence however explains that all period-3 height-periodic boundary law solutions are of the type (4.20). 
Gradient Gibbs measures for the SOS model

\section{References}

[1] M. Biskup, R. Kotecký, Phase coexistence of gradient Gibbs states, Probab. Theory Related Fields 139(1-2) (2007), 1-39. MR-2322690

[2] R. Bissacot, E.O. Endo, A.C.D. van Enter, Stability of the phase transition of critical-field Ising model on Cayley trees under inhomogeneous external fields, Stoch. Process. Appl. 127(12) (2017), 4126-4138. MR-3718108

[3] A. Bovier, Statistical mechanics of disordered systems, Cambridge University Press, Cambridge (2006). xiv+312pp. ISBN: 978-0-521-84991-3. MR-2252929

[4] A. Bovier, C. Külske, A rigorous renormalization group method for interfaces in random media, Rev. Math. Phys. 6(3) (1994), 413-496. MR-1305590

[5] A. Bovier, C. Külske, There are no nice interfaces in $2+1$ dimensional SOS-models in random media, J. Stat. Phys. 83 (1996), 751-759. MR-1386357

[6] J. Bricmont, A. Kupiainen, Phase transition in the $3 d$ random field Ising model, Comm. Math. Phys. 116(4) (1988), 539-572. MR-0943702

[7] C. Cotar, C. Külske, Existence of random gradient states, Ann. Appl. Probab. 22(4) (2012), 1650-1692. MR-2985173

[8] C. Cotar, C. Külske, Uniqueness of gradient Gibbs measures with disorder, Probab. Theory Relat. Fields 162(3) (2015), 587-635. MR-3383338

[9] J.T. Cox, Entrance laws for Markov chains, Ann. Probab. 5(3) (1977), 533-549. MR-0455128

[10] A.C.D. van Enter, C. Külske, Non-existence of random gradient Gibbs measures in continuous interface models in $d=2$, Ann. Appl. Probab. 18 (2008), 109-119. MR-2380893

[11] T. Funaki, H. Spohn, Motion by mean curvature from the Ginzburg-Landau $\nabla \varphi$ interface model, Comm. Math. Phys. 185 (1997). MR-1463032

[12] H.O. Georgii, Gibbs Measures and Phase Transitions, Second edition. De Gruyter Studies in Mathematics, 9. Walter de Gruyter, Berlin (2011). MR-2807681

[13] F.P. Kelly, Stochastic models of computer communication systems. With discussion, J. Roy. Stat. Soc. Ser. B 47 (1985), 379-395; 415-428. MR-0844469

[14] C. Külske, U.A. Rozikov, Extremality of translation-invariant phases for a three-state SOSmodel on the binary tree, J. Stat. Phys. 160(3) (2015), 659-680. MR-3366097

[15] C. Külske, P. Schriever. Gradient Gibbs measures and fuzzy transformations on trees, Markov Process. Relat. Fields 23 (2017), 553-590. MR-3754141

[16] A.E. Mazel, Y.M. Suhov, Random surfaces with two-sided constraints: an application of the theory of dominant ground states, J. Stat. Phys. 64 (1991), 111-134. MR-1117649

[17] V.V. Prasolov, Polynomials, Spinger, Berlin (2004). MR-2082772

[18] K. Ramanan, A. Sengupta, I. Ziedins, P. Mitra, Markov random field models of multicasting in tree networks, Adv. Appl. Probab. 34 (2002), 58-84. MR-1895331

[19] U.A. Rozikov, Y.M. Suhov, Gibbs measures for SOS model on a Cayley tree, Infin. Dimens. Anal. Quantum Probab. Relat. Top. 9(3) (2006), 471-488. MR-2256506

[20] U.A. Rozikov, S.A. Shoyusupov, Gibbs measures for the SOS model with four states on a Cayley tree, Theor. Math. Phys. 149(1) (2006), 1312-1323. MR-2297108

[21] U.A. Rozikov, Gibbs measures on Cayley trees, World Sci. Publ., Singapore (2013). MR3185400

[22] U.A. Rozikov, A contour method on Cayley tree, J. Stat. Phys. 130 (2008), 801-813. MR2387566

[23] S. Sheffield, Random surfaces, Am. Math. Soc. (2006). ISBN: 978-2856291870. MR-2251117

[24] S. Shlosman, Non-translation-invariant states in two dimensions, Comm. Math. Phys. 87 (1983), 497-504. MR-0691041

[25] S. Zachary, Countable state space Markov random fields and Markov chains on trees, Ann. Probab. 11(4) (1983), 894-903. MR-0714953

[26] S. Zachary, Bounded, attractive and repulsive Markov specifications on trees and on the one-dimensional lattice, Stoch. Process. Appl. 20 (1985), 559-581. MR-0808160 
Acknowledgments. U.A. Rozikov thanks the RTG 2131, the research training group on High-dimensional Phenomena in Probability - Fluctuations and Discontinuity, and the Ruhr-University Bochum (Germany) and University Paris Est Créteil (France), for financial support and hospitality.

F. Henning thanks the RTG 2131.

The research of A. Le Ny has been partly funded by the Bézout Labex, funded by ANR, reference ANR-10-LABX-58. 


\section{Electronic Journal of Probability Electronic Communications in Probability}

\section{Advantages of publishing in EJP-ECP}

- Very high standards

- Free for authors, free for readers

- Quick publication (no backlog)

- Secure publication $\left(\mathrm{LOCKSS}^{1}\right)$

- Easy interface (EJMS²)

\section{Economical model of EJP-ECP}

- Non profit, sponsored by $\mathrm{IMS}^{3}, \mathrm{BS}^{4}$, ProjectEuclid ${ }^{5}$

- Purely electronic

\section{Help keep the journal free and vigorous}

- Donate to the IMS open access fund ${ }^{6}$ (click here to donate!)

- Submit your best articles to EJP-ECP

- Choose EJP-ECP over for-profit journals

\footnotetext{
${ }^{1}$ LOCKSS: Lots of Copies Keep Stuff Safe http://www. lockss.org/

${ }^{2}$ EJMS: Electronic Journal Management System http://www.vtex.lt/en/ejms.html

${ }^{3}$ IMS: Institute of Mathematical Statistics http://www.imstat.org/

${ }^{4}$ BS: Bernoulli Society http://www. bernoulli-society.org/

${ }^{5}$ Project Euclid: https://projecteuclid.org/

${ }^{6}$ IMS Open Access Fund: http://www.imstat.org/publications/open.htm
} 